\title{
ZIV-ZAKAI BOUND FOR HARMONIC RETRIEVAL IN MULTIPLICATIVE AND ADDITIVE GAUSSIAN NOISE
}

\author{
Philippe Ciblat $^{(1)}$ and Mounir Ghogho ${ }^{(2)}$ \\ (1) Ecole Nationale Supérieure des Télécommunications (ENST), Paris, France \\ ${ }^{(2]}$ Leeds University, Leeds, United Kingdom \\ Email: philippe.ciblat@enst.fr,m.ghogho@leeds.ac.uk
}

\begin{abstract}
The paper addresses the problem of the lower performance bound evaluation for harmonic retrieval in multiplicative and additive noise, especially when the SNR is low and/or when the number of available samples is small. As a novelty, we express an accurate approximation of the Ziv-Zakai bound in closed-form. This enables us to analyse the influence of some signal parameters on performance and to characterise the so-called threshold region.
\end{abstract}

\section{INTRODUCTION}

We consider the following signal model

$$
y(n)=a(n) e^{2 i \pi\left(\varphi_{0}+\varphi_{1} n\right)}+b(n), \quad n=0, \cdots, N-1
$$

where

- $y(n)$ is the observed signal of which $N$ samples are available.

- $b(n)$ is an additive noise, which is assumed to be a circular complex-valued white Gaussian stationary process with zero-mean and variance $\sigma_{b}^{2}=\mathbb{E}\left[|b(n)|^{2}\right]$.

- $a(n)$ is a multiplicative noise, which is assumed to be a zero-mean Gaussian stationary process. This noise could be either complex or real-valued, circular or non-circular.

The parameters of interest $\varphi_{0}$ and $\varphi_{1}$ are the phase and the frequency respectively.

Such a signal model can be encountered in several application fields such as DOA estimation, radar detection and digital communications [1, 2, 3].

To characterise the lower performance bound on parameter estimation, the so-called Cramér-Rao bound (CRB) is often used, partly because of the simplicity of its derivation.

We thank the Royal Society for funding the sabbatical stay of Dr. Ciblat at Leeds University during Summer 2004
Unfortunately, it is now well-known that the CRB is not accurate at low SNR and/or when the number of samples $N$ is small. Therefore a few other bounds, which are more complicated but tighter than the CRB, have been proposed in the literature. The widely spread ones are the Barankin bound (BB) and the Ziv-Zakai bound (ZZB) $[4,5]$.

The Barankin bound for harmonic retrieval in multiplicative and additive noise has been already derived in $[6,7,8]$. Therefore, this paper focuses on the derivation and the analysis of the Ziv-Zakai bound. Our work is motivated by the fact that the Ziv-Zakai Bound appears to be tighter that the Barankin bound (of order 1), as was reported in [9, 10] in the context of additive noise models.

In the literature, different Ziv-Zakai-type bounds have been introduced and derived for the problem of bearing [11] and time-delay [12] estimation. A special case of bearing estimation involves the problem of harmonic retrieval in additive noise (without multiplicative noise). To the best of our knowledge, the derivation of the Ziv-Zakai bound when a harmonic signal is disturbed by additive and multiplicative noise sources has not been addressed in the literature. The main purpose of this paper is to fill this gap.

\section{REVIEW OF ZIV-ZAKAI BOUND}

In this section, we recall the definition of the $\mathrm{ZZB}$ in the context of a multi-variate parameter of interest.

Let $\hat{\varphi}_{0}$ and $\hat{\varphi}_{1}$ be estimates of $\varphi_{0}$ and $\varphi_{1}$ respectively. The error vector is defined as follows $\varepsilon=\left[\hat{\varphi}_{0}-\varphi_{0}, \hat{\varphi}_{1}-\right.$ $\left.\varphi_{1}\right]^{\mathrm{T}}$ where the superscript ${ }^{\mathrm{T}}$ stands for the transposition operator. Let $\varphi=\left(\varphi_{0}, \varphi_{1}\right)$. We denote the error correlation matrix by

$$
\mathbf{E}_{\varphi}=\mathbb{E}\left[\varepsilon \varepsilon^{\mathrm{T}}\right]
$$

In $[5,10]$, it was proved that the following inequality holds for any vector $\mathbf{z}$ of size $(2 \times 1)$,

$$
\mathbf{z}^{\mathrm{T}} \mathbf{E}_{\boldsymbol{\varphi}} \mathbf{z} \geq \int_{0}^{\infty} \Delta\left(\max _{\mathbf{h} \mid \mathbf{z}^{\mathrm{T}} \mathbf{h}=\Delta} f(\mathbf{h})\right) d \Delta
$$


where $\mathbf{h}=\left[h_{0}, h_{1}\right]^{\mathrm{T}}$, and

$$
f(\mathbf{h})=\int \min (p(\boldsymbol{\varphi}), p(\boldsymbol{\varphi}+\mathbf{h})) P_{e}(\boldsymbol{\varphi}, \boldsymbol{\varphi}+\mathbf{h}) d \boldsymbol{\varphi} .
$$

The function $p($.$) is the a priori density function of the$ bi-variate parameter $\boldsymbol{\varphi}$, and $P_{e}(\boldsymbol{\varphi}, \boldsymbol{\varphi}+\mathbf{h})$ is the error probability when the optimal detector (namely, the ML detector) is used to decide between the following two hypotheses

$$
\left\{\begin{array}{l}
H_{0}: y(n)=a(n) e^{2 i \pi\left(\varphi_{0}+\varphi_{1} n\right)}+b(n) \\
H_{1}: y(n)=a(n) e^{2 i \pi\left(\left(\varphi_{0}+h_{0}\right)+\left(\varphi_{1}+h_{1}\right) n\right)}+b(n)
\end{array}\right.
$$

where hypotheses $H_{0}$ and $H_{1}$ are equally likely.

The RHS of Equation (1) is called the Ziv-Zakai Bound (ZZB). Actually, it is only a (simplified) lower bound of the Ziv Zakai bound since the Valley-filling function defined in [10] is set here to be equal to the identity, and the above binary detection task is carried out under the assumption that $H_{0}$ and $H_{1}$ are equally-likely.

By inspecting Equation (1), one can remark that the likelihood of $\varphi$ is scanned over the entire search interval of $\varphi$, as is also the case for the Barankin bound [7]. This contrasts with the CRB where the likelihood function is only evaluated around the true point. Therefore the CRB is less accurate than the BB and the ZZB, especially at low SNR. Indeed at low SNR, several estimate's realizations, called "outliers", may be far away from the true point. The CRB is incapable of predicting or quantifying such phenomenon.

Since the outliers effect is particularly associated with frequency estimation, this paper focuses mainly on the estimation of $\varphi_{1}$. The ZZB for $\varphi_{1}$ is obtained by setting $\mathbf{z}=[0,1]^{\mathrm{T}}$. The mean square error for $\varphi_{1}$, denoted $\mathrm{MSE}_{1}$, is then bounded by

$$
\operatorname{MSE}_{1} \geq \int_{0}^{\infty} h_{1}\left(\max _{h_{0}} f\left(h_{0}, h_{1}\right)\right) d h_{1} .
$$

Notice that the terms $f\left(h_{0}, h_{1}\right)$ and $f(\mathbf{h})$ refer to the same function.

\section{DERIVATION OF ZIV-ZAKAI BOUND}

The key task now is to express the function $\mathbf{h} \mapsto f(\mathbf{h})$ in closed-form. According to (2), we need to explicitly express $\min (p(\boldsymbol{\varphi}), p(\boldsymbol{\varphi}+\mathbf{h}))$ and $P_{e}(\boldsymbol{\varphi}, \boldsymbol{\varphi}+\mathbf{h})$. We first focus on the derivation of the error probability $P_{e}(\varphi, \varphi+\mathbf{h})$ which can be split into two terms as follows

$$
P_{e}(\boldsymbol{\varphi}, \boldsymbol{\varphi}+\mathbf{h})=\frac{1}{2} P_{e}(\boldsymbol{\varphi} \rightarrow \boldsymbol{\varphi}+\mathbf{h})+\frac{1}{2} P_{e}(\boldsymbol{\varphi}+\mathbf{h} \rightarrow \boldsymbol{\varphi})
$$

where $P_{e}(\boldsymbol{\varphi} \rightarrow \boldsymbol{\varphi}+\mathbf{h})$ is the probability that, given the true parameter $\boldsymbol{\varphi}$ (i.e., $H_{0}$ ), the test decision is $\boldsymbol{\varphi}+\mathbf{h}$ (i.e., $H_{1}$ ), and $P_{e}(\varphi+\mathbf{h} \rightarrow \boldsymbol{\varphi})$ is defined similarly.

Before developing any further, we infer the following lemma. The proof is provided hereafter.
Lemma 1 The error probabilities $P_{e}(\varphi \rightarrow \varphi+\mathbf{h})$ and $P_{e}(\boldsymbol{\varphi}+\mathbf{h} \rightarrow \boldsymbol{\varphi})$ are identical. Furthermore, $P_{e}(\boldsymbol{\varphi}, \boldsymbol{\varphi}+\mathbf{h})$ is independent of $\varphi$.

Proof - Let $y(n)=a(n) e^{2 i \pi\left(\phi^{(0)}+\phi^{(1)} n\right)}+b(n)$ and $\phi=$ $\left[\phi^{(0)}, \phi^{(1)}\right]^{\mathrm{T}}$. The vector $\mathbf{y}=[y(0), \cdots, y(N-1)]^{\mathrm{T}}$ is then a zero-mean complex-valued Gaussian vector with correlation matrix $\mathbf{R}_{\phi}=\mathbb{E}\left[\mathbf{y} \mathbf{y}^{\mathrm{H}}\right]$ where the superscript ${ }^{\mathrm{H}}$ stands for the conjugate transposition, and with pseudo-correlation matrix $\mathbf{U}_{\phi}=\mathbb{E}\left[\mathbf{y} \mathbf{y}^{\mathrm{T}}\right]$. The matrix $\mathbf{U}_{\phi}$ is also referred to as the conjugate correlation matrix. If $\mathbf{U}_{\phi} \neq 0$, the Gaussian process is said to be non-circular or unproper.

Let $\tilde{\mathbf{y}}=\left[\mathbf{y}^{\mathrm{T}} \mathbf{y}^{\mathrm{H}}\right]^{\mathrm{T}}$. We have that

$$
\tilde{\mathbf{y}}=\tilde{\boldsymbol{\Gamma}}_{\phi} \tilde{\mathbf{a}}+\tilde{\mathbf{b}}
$$

where

$$
\tilde{\boldsymbol{\Gamma}}_{\boldsymbol{\phi}}=\left[\begin{array}{cc}
\boldsymbol{\Gamma}_{\phi} & \mathbf{0}_{N, N} \\
\mathbf{0}_{N, N} & \overline{\boldsymbol{\Gamma}_{\phi}}
\end{array}\right]
$$

with $\boldsymbol{\Gamma}_{\boldsymbol{\phi}}=\operatorname{diag}\left(e^{2 i \pi\left(\phi^{(0)}+\phi^{(1)} n\right)}, n=0, \cdots, N-1\right)$ and where $\tilde{\mathbf{a}}$ and $\tilde{\mathbf{b}}$ are defined in the same way as $\tilde{\mathbf{y}}$. As in [13], one can remark that

$$
\tilde{\mathbf{y}}=\tilde{\boldsymbol{\Gamma}}_{\phi} \tilde{\mathbf{x}}
$$

with $\tilde{\mathbf{x}}=\tilde{\mathbf{a}}+\tilde{\boldsymbol{\Gamma}}_{\phi}^{-1} \tilde{\mathbf{b}}$.

The vector $\tilde{\mathbf{X}}$ is Gaussian with zero-mean and correlation matrix $\tilde{\mathbf{R}}_{x}$ independent of $\phi$ since we have that

$$
\tilde{\mathbf{R}}_{x}=\tilde{\mathbf{R}}_{a}+\sigma_{b}^{2} \mathbf{I}_{2 N}
$$

where $\tilde{\mathbf{R}}_{a}$ the correlation matrix of $\tilde{\mathbf{a}}$ and $\mathbf{I}_{2 N}$ is the $(2 N \times$ $2 N)$ identity matrix.

By definition, $P_{e}(\boldsymbol{\varphi} \rightarrow \boldsymbol{\varphi}+\mathbf{h})$, which corresponds to the error probability of the ML detector, can be written as follows

$$
P_{e}(\boldsymbol{\varphi} \rightarrow \boldsymbol{\varphi}+\mathbf{h})=\operatorname{Prob}\left(\frac{p(\mathbf{y} \mid \boldsymbol{\varphi}+\mathbf{h})}{p(\mathbf{y} \mid \boldsymbol{\varphi})}>1 \mid \tilde{\mathbf{y}}=\tilde{\boldsymbol{\Gamma}}_{\boldsymbol{\varphi}} \tilde{\mathbf{x}}\right)
$$

Since $\mathbf{y}$ is Gaussian, the likelihood function is given by

$$
p(\mathbf{y} \mid \phi) \propto \frac{1}{\sqrt{\left|\operatorname{det}\left(\tilde{\mathbf{R}}_{\boldsymbol{\phi}}\right)\right|}} e^{-\frac{1}{2} \tilde{\mathbf{y}}^{\mathrm{H}} \tilde{\mathbf{R}}_{\phi}^{-1} \tilde{\mathbf{y}}}
$$

where

$$
\begin{aligned}
\tilde{\mathbf{R}}_{\phi} & :=\mathbb{E}\left[\tilde{\mathbf{y}} \tilde{\mathbf{y}}^{\mathrm{H}}\right] \\
& =\left[\begin{array}{ll}
\mathbf{R}_{\phi} & \mathbf{U}_{\phi} \\
\overline{\mathbf{U}_{\phi}} & \overline{\mathbf{R}_{\phi}}
\end{array}\right]
\end{aligned}
$$

and where the overline stands for the complex conjugate operator. According to Eq. (3), we have that

$$
\tilde{\mathbf{R}}_{\phi}=\tilde{\boldsymbol{\Gamma}}_{\phi} \tilde{\mathbf{R}}_{x} \tilde{\boldsymbol{\Gamma}}_{\phi}^{\mathrm{H}}
$$


which implies that $\operatorname{det}\left(\tilde{\mathbf{R}}_{\phi}\right)$ is independent of $\phi$.

Consequently

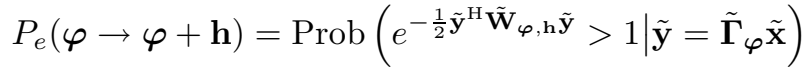

with

$$
\tilde{\mathbf{W}}_{\boldsymbol{\varphi}, \mathbf{h}}=\tilde{\mathbf{R}}_{\boldsymbol{\varphi}+\mathbf{h}}^{-1}-\tilde{\mathbf{R}}_{\boldsymbol{\varphi}}^{-1}
$$

Using $\tilde{\mathbf{y}}=\tilde{\boldsymbol{\Gamma}}_{\varphi} \tilde{\mathbf{x}}$ and Eq. (4), we obtain

$$
P_{e}(\boldsymbol{\varphi} \rightarrow \boldsymbol{\varphi}+\mathbf{h})=\operatorname{Prob}\left(e^{-\frac{1}{2} \tilde{\mathbf{x}}^{\mathrm{H}} \tilde{\mathbf{W}}_{\mathbf{h}} \tilde{\mathbf{x}}}>1\right)
$$

with

$$
\begin{aligned}
\tilde{\mathbf{W}}_{\mathbf{h}} & :=\tilde{\mathbf{W}}_{\mathbf{0}, \mathbf{h}} \\
& =\tilde{\mathbf{R}}_{\mathbf{h}}^{-1}-\tilde{\mathbf{R}}_{\mathbf{0}}^{-1} \\
& =\tilde{\boldsymbol{\Gamma}}_{\mathbf{h}} \tilde{\mathbf{R}}_{x}^{-1} \tilde{\boldsymbol{\Gamma}}_{\mathbf{h}}^{\mathrm{H}}-\tilde{\mathbf{R}}_{x}^{-1} .
\end{aligned}
$$

Thus, $P_{e}(\varphi \rightarrow \varphi+\mathbf{h})$ is independent of $\varphi$ since the statistics of $\tilde{\mathbf{x}}$ are independent of $\varphi$. Using the same reasoning as above, $P_{e}(\boldsymbol{\varphi}+\mathbf{h} \rightarrow \boldsymbol{\varphi})$ can be shown to be given by the same expression as in the RHS of eq. (5). This concludes the proof.

Thanks to Lemma 1 , we are now able to simplify $f(\mathbf{h})$ as follows [10]

$$
f(\mathbf{h})=A(\mathbf{h}) P_{e}(\mathbf{h})
$$

where

$$
A(\mathbf{h})=\int \min (p(\boldsymbol{\varphi}), p(\boldsymbol{\varphi}+\mathbf{h})) d \boldsymbol{\varphi}
$$

and $P_{e}(\mathbf{h})$ stands for $P_{e}(\varphi, \varphi+\mathbf{h})$. Next, we derive $A(\mathbf{h})$ and $P_{e}(\mathbf{h})$.

Since we have no a priori information on $\varphi$, we consider that $\varphi_{0}$ and $\varphi_{1}$ are uniformly distributed over $[0,1 / 2]$, i.e. the a priori distribution of the parameters of interest $p(\boldsymbol{\varphi})$ is flat. The upper bound $1 / 2$ is chosen since the phase and the frequency can only be estimated modulo $1 / 2$ when multiplicative noise occurs [10]. Consequently

$$
A(\mathbf{h})=\left(1 / 2-h_{0}\right)\left(1 / 2-h_{1}\right)
$$

This leads to

$\mathrm{MSE}_{1} \geq \int_{0}^{1 / 2}\left(1 / 2-h_{1}\right) h_{1}\left(\max _{h_{0}}\left(1 / 2-h_{0}\right) P_{e}\left(h_{0}, h_{1}\right)\right) d h_{1}$ where $P_{e}\left(h_{0}, h_{1}\right)$ represents the same function as $P_{e}(\mathbf{h})$.

The rest of the paper deals with the evaluation of $P_{e}(\mathbf{h})$. According to Eq. (5), we have that

$$
P_{e}(\mathbf{h})=\operatorname{Prob}\left(\tilde{\mathbf{x}}^{\mathrm{H}} \tilde{\mathbf{W}}_{\mathbf{h}} \tilde{\mathbf{x}}<0\right)
$$

In words, we wish to calculate the probability that a quadratic form, which is neither positive nor negative, of a Gaussian vector is negative.
Firstly, we change the complex-valued vector $\tilde{\mathbf{x}}$ into its associated real-valued vector $\breve{\mathbf{x}}=\left[\Re\left(\mathbf{x}^{\mathrm{T}}\right), \Im\left(\mathbf{x}^{\mathrm{T}}\right)\right]^{\mathrm{T}}$ where $\Re($.$) and \Im($.$) are the real and imaginary part of a complex-$ valued number respectively. Consequently, Eq. (6) becomes

$$
P_{e}(\mathbf{h})=\operatorname{Prob}\left(\breve{\mathbf{x}}^{\mathrm{T}} \breve{\mathbf{W}}_{\mathbf{h}} \breve{\mathbf{x}}<0\right)
$$

with

$$
\breve{\mathbf{W}}_{\mathbf{h}}=\breve{\mathbf{R}}_{\mathbf{h}}^{-1}-\breve{\mathbf{R}}_{\mathbf{0}}^{-1}
$$

and

$$
\breve{\mathbf{R}}_{\phi}=\mathbb{E}\left[\breve{\mathbf{y}} \breve{\mathbf{y}}^{\mathrm{T}}\right] .
$$

Secondly, in order to obtain a simple expression for $P_{e}(\mathbf{h})$, we proceed by whitening the process $\breve{\mathbf{x}}$. This is achieved by diagonalising $\breve{\mathbf{R}}_{x}:=\mathbb{E}\left[\breve{\mathbf{x}} \breve{\mathbf{x}}^{\mathrm{T}}\right]$. According to Eq. (4), we prove that $\breve{\mathbf{R}}_{\mathbf{0}}=\breve{\mathbf{R}}_{x}$ where $\breve{\mathbf{R}}_{\mathbf{0}}=\breve{\mathbf{R}}_{\phi_{\mid \phi=0}}$. This matrix can be decomposed as follows

$$
\breve{\mathbf{R}}_{\mathbf{0}}=\mathbf{D}_{0}^{\mathrm{T}} \boldsymbol{\Lambda}_{0} \mathbf{D}_{0}
$$

where $\mathbf{D}_{0}$ is an orthogonal real-valued matrix of size $(2 N \times$ $2 N)$ and $\boldsymbol{\Lambda}_{0}$ is a positive real-valued diagonal matrix. Therefore, the multi-variate random variable $\breve{\mathbf{z}}=\boldsymbol{\Lambda}_{0}^{-1 / 2} \mathbf{D}_{0} \breve{\mathbf{x}}$ is a white Gaussian vector.

Thirdly, we concentrate on the term $\breve{\mathbf{x}}^{\mathrm{T}} \breve{\mathbf{W}}_{\mathbf{h}} \breve{\mathbf{x}}$. Thanks to the decomposition of $\breve{\mathbf{R}}_{\mathbf{0}}$, we get

$$
\breve{\mathbf{x}}^{\mathrm{T}} \breve{\mathbf{W}}_{\mathbf{h}} \breve{\mathbf{x}}=\breve{\mathbf{z}}^{\mathrm{T}} \mathbf{V} \breve{\mathbf{z}}
$$

with

$$
\mathbf{V}_{\mathbf{h}}=\boldsymbol{\Lambda}_{0}^{1 / 2} \mathbf{D}_{0} \breve{\mathbf{W}}_{\mathbf{h}} \mathbf{D}_{0}^{\mathrm{T}} \boldsymbol{\Lambda}_{0}^{1 / 2}
$$

One can observe that $\mathbf{V}_{\mathbf{h}}$ is a symmetric matrix which can be decomposed as

$$
\mathbf{V}_{\mathbf{h}}=\mathbf{D}_{\mathbf{h}}^{\mathrm{T}} \boldsymbol{\Lambda}_{\mathbf{h}} \mathbf{D}_{\mathbf{h}}
$$

where $\mathbf{D}_{\mathbf{h}}$ is an orthogonal real-valued matrix of size $2 N \times$ $2 N$ and $\boldsymbol{\Lambda}_{\mathbf{h}}$ is a diagonal real-valued matrix. Notice that the matrix $\boldsymbol{\Lambda}_{\mathbf{h}}$ is neither positive nor negative. Therefore the set of its eigenvalues can be split into two parts: the positive ones and the negatives ones. We consider that the first $m$ eigenvalues are negative and are denoted by $-\lambda_{n}^{(2)}$ with $\lambda_{n}^{(2)}>0$ for $n=0, \cdots, m-1$. The positive or null eigenvalues are denoted $\lambda_{n}^{(1)}$ with $\lambda_{n}^{(1)} \geq 0$ for $n=$ $m, \cdots, 2 N-1$. Thus, we get

$$
\boldsymbol{\Lambda}_{\mathbf{h}}=\operatorname{diag}\left(-\lambda_{0}^{(2)}, \cdots,-\lambda_{m-1}^{(2)}, \lambda_{m}^{(1)}, \cdots, \lambda_{2 N-1}^{(1)}\right) .
$$

Recall that these eigenvalues depend on $\mathbf{h}$.

Let $\mathbf{u}=\left[u_{0}, \cdots, u_{2 N-1}\right]^{\mathrm{T}}=\mathbf{D}_{\mathbf{h}} \breve{\mathbf{z}}$ be a zero-mean unit-variance Gaussian vector. By construction, $\mathbf{u}$ is white, so its statistics do not depend on $\mathbf{h}$. By replacing $\breve{\mathbf{z}}$ and $\mathbf{V}_{\mathbf{h}}$ with $\mathbf{u}$ and Eq. (8) respectively, we obtain

$$
\breve{\mathbf{x}}^{\mathrm{T}} \breve{\mathbf{W}}_{\mathbf{h}} \breve{\mathbf{x}}=\mathbf{u}^{\mathrm{T}} \boldsymbol{\Lambda}_{\mathbf{h}} \mathbf{u} .
$$


Finally, we obtain

$$
P_{e}(\mathbf{h})=\operatorname{Prob}\left(\sum_{n=m}^{2 N-1} \lambda_{n}^{(1)} u_{n}^{2}<\sum_{n=0}^{m-1} \lambda_{n}^{(2)} u_{n}^{2}\right) .
$$

where $\left\{u_{n}\right\}$ is a real-valued i.i.d. Gaussian random process with zero-mean and unit-variance.

We now wish to derive a closed-form expression for the following term

$$
P_{e}(\mathbf{h})=\operatorname{Prob}\left(p_{1}<p_{2}\right)
$$

where $p_{m}=\sum_{n} \lambda_{n}^{(m)} u_{n}^{2}$ is a weighted sum of squared independent Gaussian variable. Notice that, by construction, $p_{1}$ and $p_{2}$ are independent.

If $\lambda_{n}=\lambda$ for all $n$, then $p_{1}$ (resp. $p_{2}$ ) obeys a $\chi^{2}$ distribution with $(2 N-m)$ (resp. $m$ ) degrees of freedom. However, if the weighting coefficients are different, the $p_{m}$ 's are not $\chi^{2}$ distributed anymore. Further, expressing the distribution of $p_{m}$ in closed-form is not tractable. Nevertheless, it can be well approximated by means of the Gamma distribution [14]. We recall that the Gamma distribution, denoted $\mathcal{G}(\alpha, \theta)$, is defined as follows

$$
P_{\alpha, \theta}(x)=\frac{x^{\alpha-1}}{\Gamma(\alpha) \theta^{\alpha}} e^{-x / \theta}
$$

where $\Gamma($.$) is the so-called Gamma function.$

Hence, the distribution of $p_{m}$ is next approximated by the Gamma distribution whose first and second moments are equal to those of $p_{m}$. We thus obtain

$$
p_{1} \sim \mathcal{G}\left(\alpha_{1}, \theta_{1}\right) \quad \text { and } \quad p_{2} \sim \mathcal{G}\left(\alpha_{2}, \theta_{2}\right)
$$

with

$$
\alpha_{1}=\frac{1}{2} \frac{\left(\sum_{n=m}^{2 N-1} \lambda_{n}^{(1)}\right)^{2}}{\sum_{n=m}^{2 N-1} \lambda_{n}^{(1)^{2}}} \text { and } \theta_{1}=2 \frac{\sum_{n=m}^{2 N-1} \lambda_{n}^{(1)}{ }^{2}}{\sum_{n=m}^{2 N-1} \lambda_{n}^{(1)}},
$$

and

$$
\alpha_{2}=\frac{1}{2} \frac{\left(\sum_{n=0}^{m-1} \lambda_{n}^{(2)}\right)^{2}}{\sum_{n=0}^{m-1} \lambda_{n}^{(2)^{2}}} \text { and } \theta_{2}=2 \frac{\sum_{n=0}^{m-1} \lambda_{n}^{(2)}}{\sum_{n=0}^{m-1} \lambda_{n}^{(2)}} .
$$

As $p_{m}$ is now assumed Gamma distributed, Eq. (10) can be simplified. Indeed, by using the fact that the square root of a Gamma distributed random variable is Nakagami distributed, and then by using Eq. (46) in [15], we have that

$$
P_{e}(\mathbf{h})=\frac{\theta^{\alpha_{1}}}{\alpha_{1}} B\left(\alpha_{1}, \alpha_{2}\right)_{2} F_{1}\left(\alpha_{1}+\alpha_{2}, \alpha_{1}, \alpha_{1}+1 ;-\theta\right)
$$

where

$$
\text { - } \theta=\theta_{1} / \theta_{2} \text {, }
$$

- $B\left(\alpha_{1}, \alpha_{2}\right)=\Gamma\left(\alpha_{1}+\alpha_{2}\right) / \Gamma\left(\alpha_{1}\right)$ is called either the Euler's first integral or the Beta function,

- ${ }_{2} F_{1}(\alpha, \beta, \gamma ; x)$ is the so-called hyper-geometric function.

The above expression for $P_{e}(\mathbf{h})$ represents the main result of this paper. Although this expression is not interpretable, its numerical computation will provide interesting results.

\section{SIMULATIONS}

The multiplicative noise $a(n)$ is hereafter assumed to be white non-circular Gaussian process with zero-mean, unitvariance, and pseudo-variance $\rho=\mathbb{E}\left[a(n)^{2}\right]$. For sake of simplicity, we also assume that the real part of $a(n)$ is independent of its imaginary part. This implies that $\rho$ is realvalued. If $\rho=0$, then $a(n)$ is circular; if $\rho=1$, then $a(n)$ is real-valued. Thus, $\rho$ quantifies the non-circularity rate of $a(n)$. In each figure, we display four curves: dashed line corresponds to the empirical mean square error (MSE) for the well-known NLS estimate $[1,2,16]$. Solid lines with star point represent the ZZB. Solid lines with triangular point and with circle point plot represent the BB and the CRB respectively [16, 7].

In Figure 1, we plot all the curves versus SNR with $N=64, \rho=1$. We observe that the ZZB is significantly tighter than the BB. The SNR threshold value predicted by the ZZB is quite close to that obtained empirically with the NLS estimate.

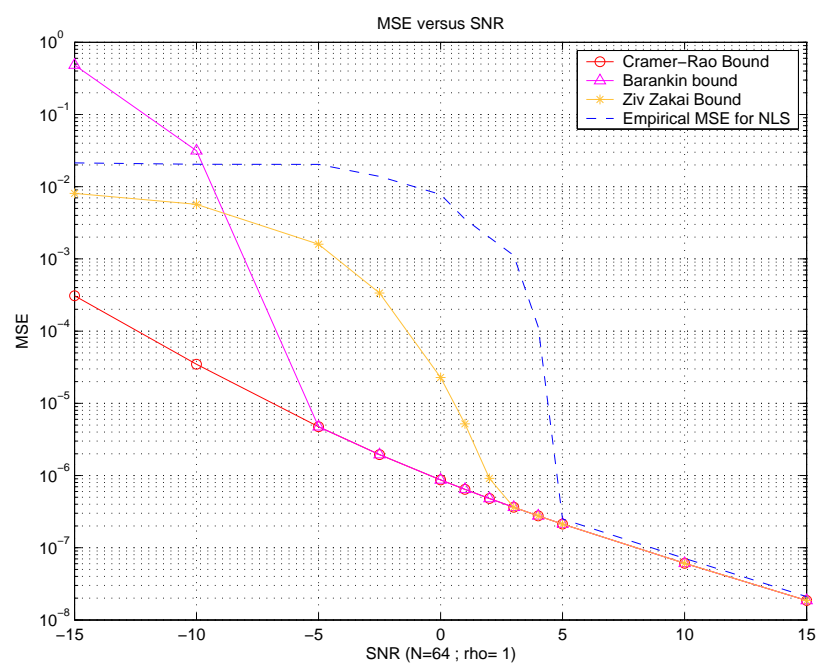

Fig. 1. MSE versus SNR

In Figure 2, we plot the curves versus $N$ with SNR $=$ $10 \mathrm{~dB}, \rho=0.9$. Even though the ZZB offers a more realistic value for the $N$ threshold than the BB, the mismatch 
between the ZZB and the NLS performance is still quite large.

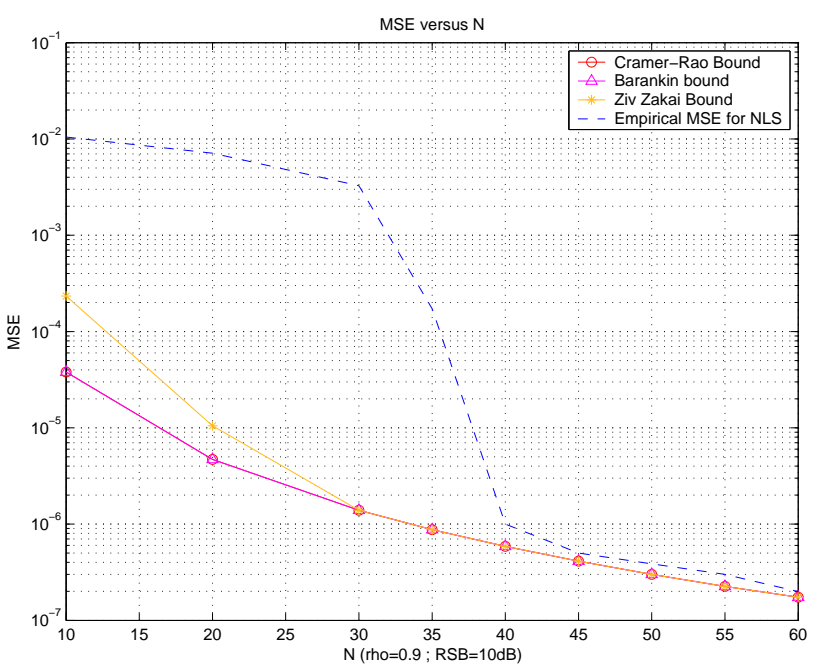

Fig. 2. MSE versus $N$

In Figure 3, the curves are displayed versus $\rho$ with $N=$ $64, \mathrm{SNR}=10 \mathrm{~dB}$. The figure confirms that accurate frequency estimation is really difficult to achieve when the signal is not non-circular enough.

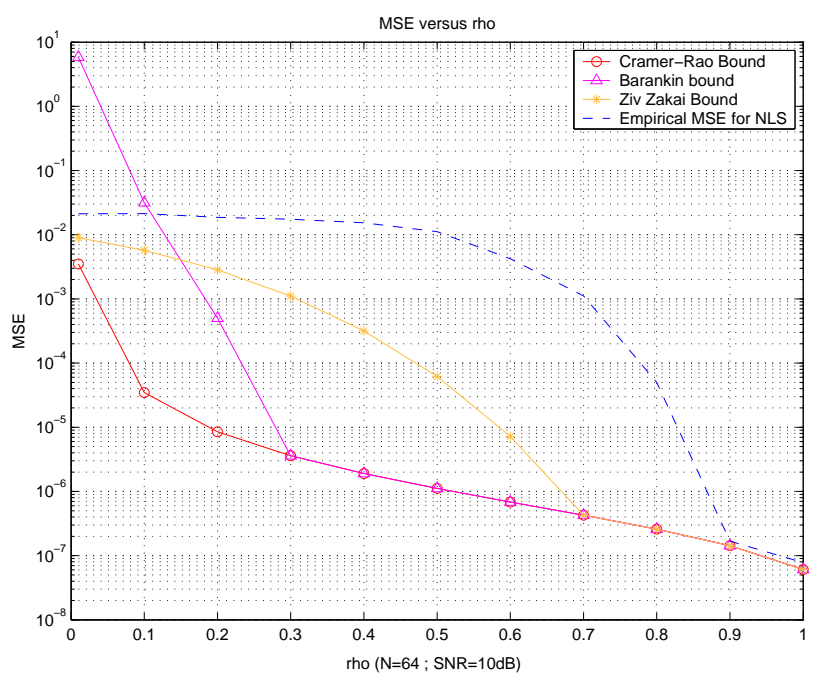

Fig. 3. MSE versus $\rho$

\section{CONCLUSION}

This paper developed an approximate closed-form expressions for the Ziv-Zakai bound on frequency estimation in the context of both additive and multiplicative noise. These expressions were used in an attempt to shed some light on the threshold effect problem associated with low SNR, small number of samples, or weak non-circularity of the multiplicative noise.

\section{ACKNOWLEDGEMENT}

The authors would like to thank Dr. Debarge (ENST Paris) for his gracious mathematical help.

\section{REFERENCES}

[1] O. Besson and P. Stoica, "Nonlinear least-squares approach to frequency estimation and detection for sinusoidal signals with arbitrary envelope," Digital Signal Processing, vol. 9, no. 1, pp. 45-56, Jan. 1999.

[2] M. Ghogho, A. Swami, and A.K. Nandi, "Non-linear least squares estimation for harmonics in multiplicative and additive noise," Signal Processing, pp. 43-60, Oct. 1999.

[3] M. Ghogho, A. Swami, and T.S. Durrani, "Frequency estimation in the presence of Doppler spread : performance analysis," IEEE Trans. on Signal Processing, vol. 49, no. 4, pp. 777-789, Apr. 2001.

[4] E.W. Barankin, "Locally best unbiaised estimates," Annals of Mathematical Statistics, vol. 20, pp. 447501, 1949.

[5] J. Ziv and M. Zakai, "Some lower bounds on Signal Processing estimation," IEEE Trans. on Information Theory, vol. 15, no. 3, pp. 386-391, May 1969.

[6] H. Messer, "Source localization performance and the array beampattern," Signal Processing, vol. 28, no. 2, pp. 163-181, Aug. 1992.

[7] P. Ciblat, P. Forster, and P. Larzabal, "Harmonic retrieval in non-circular complex-valued multiplicative noise : Barankin bound," in European Signal Processing Conference (EUSIPCO), 2004.

[8] P. Ciblat, M. Ghogho, P. Larzabal, and P. Forster, "Harmonic retrieval in the presence of non-circular gaussian multiplicative noise : Performance bounds," EURASIP Signal Processing, vol. 85, no. 4, pp. 737749, Apr. 2005.

[9] D. Chazan, M. Zakai, and J. Ziv, "Improved lower bounds on Signal Processing estimation," IEEE Trans. on Information Theory, vol. 21, pp. 90-93, Jan. 1975. 
[10] K.L. Bell, Y. Steinberg, Y. Ephraim, and H.L. Van Trees, "Extended Ziv-Zakai lower bound for vector parameter estimation," IEEE Trans. on Information Theory, vol. 43, no. 2, pp. 624-637, Mar. 1997.

[11] K.L. Bell, Y. Ephraim, and H.L. Van Trees, "Explicit Ziv-Zakai lower bound for Bearing estimation," IEEE Trans. on Signal Processing, vol. 44, no. 11, pp. 28102824, Nov. 1996.

[12] A. Weiss and E. Weinstein, "Fundamental limitations in passive time-delay estimation - Part I: Narrow-band systems," IEEE Trans. on Acoustics, Speech, and Signal Processing, vol. 31, no. 2, pp. 472-486, Apr. 1983.

[13] M. Ghogho, A.K. Nandi, and A. Swami, "CramerRao bounds and maximum likelihood estimation for random amplitude phase-modulated signals," IEEE Trans. on Signal Processing, vol. 47, no. 11, pp. 29052916, Nov. 1999.
[14] Q. Zhang and D. Liu, "A simple capacity formula for correlated diversity Rician fading channels," IEEE Communications Letters, vol. 6, no. 11, pp. 481-483, Nov. 2002.

[15] M.K. Simon and M.S. Alouini, "On the distribution of two Chi-square variates with application to outage probability computation," IEEE Trans. on Communications, vol. 49, no. 11, pp. 1946-1954, Nov. 2001.

[16] P. Ciblat and M. Ghogho, "Harmonic retrieval in non-circular complex-valued multiplicative noise : Cramer-Rao bound," in International Confererence on Acoustics, Speech, and Signal Processing (ICASSP), 2004. 